\title{
Analysis and Control of the Complex Dynamics of a Multimarket Cournot Investment Game with Bounded Rationality
}

\author{
LiuWei Zhao \\ Computational Experiment Center for Social Science, School of Management, Jiangsu University, Zhenjiang, Jiangsu 212013, China \\ Correspondence should be addressed to LiuWei Zhao; 136901672@qq.com
}

Received 22 February 2016; Revised 29 March 2016; Accepted 7 April 2016

Academic Editor: Christos K. Volos

Copyright (c) 2016 LiuWei Zhao. This is an open access article distributed under the Creative Commons Attribution License, which permits unrestricted use, distribution, and reproduction in any medium, provided the original work is properly cited.

\begin{abstract}
A dynamic multimarket Cournot model is introduced based on a specific inverse demand function. Puu's incomplete information approach, as a realistic method, is used to contract the corresponding dynamical model under this function. Therefore, some stability analysis is carried out on the model to detect the stability and instability conditions of the system's Nash equilibrium. Based on the analysis, some dynamic phenomena such as bifurcation and chaos are found. Numerical simulations are used to provide experimental evidence for the complicated behaviors of the system evolution. It is observed that the equilibrium of the system can lose stability via flip bifurcation or Neimark-Sacker bifurcation and time-delayed feedback control is used to stabilize the chaotic behaviors of the system.
\end{abstract}

\section{Introduction}

The dynamical behaviors of oligopoly games are complex because every oligopolistic producer in each period must consider not only its own decision but also the reactions of all other competitors. Cournot competition is an economic model used to describe the competition between some companies on the amount of output they will produce [1]. Thus a generalization of this game to the case of two markets is done. It is shown that the resulting dynamics is quite variable. In the classic model, each participant uses a naive expectation to suppose that the opponents' output keeps the same level as the previous period and adopts an output strategy to maximize the expected profit. Many researchers have analyzed the system stability and the complex phenomena in Cournot oligopoly games with this kind of expectation [2-9]. In an early work [10], a kind of bounded rationality is assumed for the dynamical Cournot game, where each producer does not have complete knowledge of the market and updates its production by the local profit maximization method.

In recent years, a great amount of work has been done on the dynamical Cournot games with homogeneous or heterogeneous expectations. Bounded rationality in the marginal profit method is assumed to all producers in the models considering homogeneous expectation [10-13]. The models with heterogeneous expectations (naïve, boundedly rational, or adaptive) have been discussed in many other works [1420].

In this study, a dynamic multimarket Cournot model is introduced based on a specific inverse demand function. The main purpose of our work is to formulate a novel model, which puts investment decision as a substitute for output adjustment into the dynamical Cournot game. In the model, all producers are also assumed to have bounded rationality and make their investment decisions in line with the marginal profit in the previous period. Meanwhile each firm will increase its investment if it perceives a growing marginal profit and decreases its investment if the perceived marginal profit is decreased. During a local adjustment process, this novel dynamical Cournot game aims to develop the equilibrium or demonstrate complex dynamic behaviors.

This paper is organized as follows. In Section 2, we model the dynamical game played by players with bounded rationality. In Section 3, we discuss the existence and local stability of the equilibrium points for the system. In Section 4, we show the dynamic features of this system with numerical simulations, including bifurcation diagram, phase portrait, and sensitive dependence on initial conditions. In Section 5, time-delayed feedback control is used to stabilize the chaotic behaviors of the system. 


\section{The Multimarket Cournot Model}

In this model, the products are near substitutes but different in the quality levels. So, firms can charge different prices for different markets. Suppose we have $n$ firms $(n>2)$ that compete in two interrelated markets $A$ and $B$. For the price in the market, we consider Bowley [21] who has introduced the demand function

$$
p_{i}=\psi_{i}-q_{i}-\theta \sum_{j \neq i} q_{j}
$$

where $0 \leq \theta \leq 1$. We also suppose that the production cost function of each firm takes a specific inverse demand function; [22-24] used this form in their work. Billand et al. [25] used the same demand function with $\theta=1$. Let the demand in market $A$ for firm $i$ be

$$
p_{A i}=\psi_{i}-a_{i}\left(q_{A i}+\sum_{j \neq i} q_{A j}\right),
$$

where $p_{A i}$ is the firm $i$ 's price in markets $A, q_{A i}$ is the firm $i$ 's quantity and the constant $\psi_{i}>0, a_{i}>0$ for market $A$. The demand in market $B$ for firm $i$ is

$$
p_{B i}=\beta_{i}-b_{i}\left(q_{B i}+\sum_{j \neq i} q_{B j}\right),
$$

where $p_{B i}$ is the firm $i$ 's price in markets $B, q_{B i}$ is the firm $i$ 's quantity and the constant $\beta_{i}>0, b_{i}>0$ for market $B$.

The cost function of the firm is

$$
C_{i}\left(q_{A i}, q_{B i}\right)=c_{i}\left(q_{A i}+q_{B i}\right)^{2} .
$$

Hence the profit of firm $i$ is given by

$$
\pi_{i}=p_{A i} q_{A i}+p_{B i} q_{B i}-C_{i}\left(q_{A i}+q_{B i}\right)^{2} .
$$

A standard approach to generalize static games to dynamic ones is called the bounded rationality approach $[3,5]$. The corresponding dynamical system for the static multimarket Cournot model is

$$
\begin{aligned}
q_{A i}(t+1)=q_{A i}(t)+\gamma_{A i}\left(q_{A i}(t)\right) \frac{\partial \pi_{i}}{\partial q_{A i}(t)} & \\
i=1,2, \ldots, n, & \\
q_{B i}(t+1)=q_{B i}(t)+\gamma_{B i}\left(q_{B i}(t)\right) \frac{\partial \pi_{i}}{\partial q_{B i}(t)}, & \\
i & =1,2, \ldots, n .
\end{aligned}
$$

Here, we study the monopoly case. In this case there is only one firm $(n=1)$ and two markets. We use the speeds (rates) of adjustment as linear functions of the quantities. For instance, we take $\gamma_{A i}\left(q_{A i}(t)\right)=\gamma_{A}\left(q_{A i}(t)\right)$ and $\gamma_{B i}\left(q_{B i}(t)\right)=$ $\gamma_{B}\left(q_{B i}(t)\right)$ as an example, where $\gamma_{A}$ and $\gamma_{B}$ are constants, $a_{1}=$ $a, \psi_{1}=\psi, \beta_{1}=\beta$, and $b_{1}=b$. With all the assumptions above, we get firm profit in period $t$ as follows:

$$
\begin{aligned}
\pi\left(q_{A}, q_{B}\right)= & q_{A}\left[\psi-a q_{A}\right]+q_{B}\left[\beta-b q_{B}\right] \\
& -c\left(q_{A}+q_{B}\right)^{2} .
\end{aligned}
$$

By differentiating $\pi\left(q_{A}, q_{B}\right)$, we obtain firm marginal profit with respect to its investment in period $t$ at the markets $A$ and $B$, respectively, as follows:

$$
\begin{aligned}
& \frac{\partial \pi}{\partial q_{A}}=\psi-2 q_{A}(a+c)-2 c q_{B} \\
& \frac{\partial \pi}{\partial q_{B}}=\beta-2 q_{B}(b+c)-2 c q_{A} .
\end{aligned}
$$

According to the theory of Ding [26], hence, we get the dynamic system as follows:

$$
\begin{aligned}
& q_{A}(t+1)=q_{A}(t)+\gamma_{A} q_{A}(t) \\
& \cdot\left[\omega\left(\psi-2(a+c) q_{A}(t)-2 c q_{B}(t)\right)\right. \\
& \left.+(1-\omega)\left(\psi-2(a+c) q_{A}(t-1)-2 c q_{B}(t-1)\right)\right] \\
& q_{B}(t+1)=q_{B}(t)+\gamma_{B} q_{B}(t) \\
& \cdot\left[\omega\left(\beta-2(b+c) q_{B}(t)-2 c q_{A}(t)\right)\right. \\
& \left.+(1-\omega)\left(\beta-2(b+c) q_{B}(t-1)-2 c q_{A}(t-1)\right)\right],
\end{aligned}
$$

where $0 \leq w \leq 1$ is a weight coefficient assigned to the nondelayed period $t$ and $1-w$ is assigned to the delayed period $t-1$.

Let $I_{1}(t)=q_{A}(t-1), I_{2}(t)=q_{B}(t-1)$; we obtain a fourdimensional discrete dynamic system:

$$
\begin{aligned}
& q_{A}(t+1)=q_{A}(t)+\gamma_{A} q_{A}(t) \\
& \cdot\left[\omega\left(\psi-2(a+c) q_{A}(t)-2 c q_{B}(t)\right)\right. \\
& \left.+(1-\omega)\left(\psi-2(a+c) I_{1}(t)-2 c I_{2}(t)\right)\right] \\
& q_{B}(t+1)=q_{B}(t)+\gamma_{B} q_{B}(t) \\
& \cdot\left[\omega\left(\beta-2(b+c) q_{B}(t)-2 c q_{A}(t)\right)\right. \\
& \left.\quad+(1-\omega)\left(\beta-2(b+c) I_{2}(t)-2 c I_{1}(t)\right)\right] \\
& I_{1}(t+1)=q_{A}(t) \\
& I_{2}(t+1)=q_{B}(t) .
\end{aligned}
$$

System (10) describes a duopoly game played by boundedly rational players making decision in a process of dynamical investment in the two markets. In the following sections, we are to investigate the dynamical properties of this model. 


\section{Analysis of the Equilibrium Points and Stability}

Let $q_{\xi}(t+1)=q_{\xi}(t)$ and $I_{\xi}(t+1)=I_{\xi}(t)(\xi=A, B)$ in system (10); then we get

$$
\begin{aligned}
& q_{A}(t)\left[\omega\left(\psi-2(a+c) q_{A}(t)-2 c q_{B}(t)\right)\right. \\
& \left.+(1-\omega)\left(\psi-2(a+c) I_{1}(t)-2 c I_{2}(t)\right)\right]=0 \\
& q_{B}(t)\left[\omega\left(\beta-2(b+c) q_{B}(t)-2 c q_{A}(t)\right)\right. \\
& \left.+(1-\omega)\left(\beta-2(b+c) I_{2}(t)-2 c I_{1}(t)\right)\right]=0 \\
& I_{1}(t)=q_{A}(t) \\
& I_{2}(t)=q_{B}(t) .
\end{aligned}
$$

Solving equations in (11), we obtain four equilibrium states of dynamics (see (10)), which are listed as follows:

$$
\begin{aligned}
& E_{0}=(0,0,0,0), \\
& E_{1}=\left(0, \frac{\beta}{2(b+c)}, 0, \frac{\beta}{2(b+c)}\right), \\
& E_{2}=\left(\frac{\psi}{2(b+c)}, 0, \frac{\psi}{2(b+c)}, 0\right),
\end{aligned}
$$

$$
\begin{aligned}
E^{*} & =\left(q_{A}^{*}, q_{B}^{*}, I_{1}^{*}, I_{2}^{*}\right)=\left(\frac{-\beta c+\psi b+c \psi}{2(b c+a b+a c)},\right. \\
& \frac{a \beta+\beta c-\psi c}{2(b c+a b+a c)}, \frac{-\beta c+\psi b+c \psi}{2(b c+a b+a c)}, \\
& \left.\frac{a \beta+\beta c-\psi c}{2(b c+a b+a c)}\right) .
\end{aligned}
$$

In order to make these equilibrium points have economic meaning, we only consider the nonnegative cases. Since $\beta, \psi$, $b$, and $c$ are positive parameters, $E_{1}, E_{2}$, and $E^{*}$ are all positive provided that

$$
\begin{aligned}
& \psi b+c \psi-\beta c>0, \\
& a \beta+\beta c-\psi c>0 .
\end{aligned}
$$

In the following, all the nonnegativity conditions (13a) and (13b) are assumed. $E_{0}, E_{1}$, and $E_{2}$ are all boundary equilibriums and $E^{*}$ is a unique interior equilibrium. Next, we will analyze the stability of the equilibrium.

3.1. Stability of the Boundary Equilibriums. To investigate the local stability of an equilibrium $\left(q_{A}, q_{B}, I_{1}, I_{2}\right)$ of system (9), we work out its Jacobian matrix $J$ :

$$
J\left(q_{A}, q_{B}, I_{1}, I_{2}\right)=\left(\begin{array}{cccc}
1+\gamma_{A} N_{1} & -2 c \omega \gamma_{A} q_{A 1}(t) & -2(1-\omega)(a+c) \gamma_{A} q_{A 1}(t) & -2 c \gamma_{A} q_{A 1}(t)(1-\omega) \\
-2 \omega c \gamma_{B} q_{B 1}(t) & 1+\gamma_{B} N_{2} & -2 c \gamma_{B} q_{B 1}(t)(1-\omega) & -2(b+c) \gamma_{B} q_{B 1}(t)(1-\omega) \\
1 & 0 & 0 & 0
\end{array}\right)
$$

where

$$
\begin{aligned}
N_{1}= & \omega\left(\psi-4(a+c) q_{A 1}(t)-2 c q_{B 1}(t)\right) \\
& +(1-\omega)\left(\psi-2(a+c) I_{1}(t)-2 c I_{2}(t)\right), \\
N_{2}= & \omega\left(\beta-4(b+c) q_{B 1}(t)-2 c q_{A 1}(t)\right) \\
& +(1-\omega)\left(\beta-2(b+c) I_{2}(t)-2 c I_{1}(t)\right) .
\end{aligned}
$$

An equilibrium $\left(q_{A}, q_{B}, I_{1}, I_{2}\right)$ will be locally asymptotically stable if all the eigenvalues (real or complex) of the Jacobian matrix $J\left(q_{A}, q_{B}, I_{1}, I_{2}\right)$ lie inside the unit disk; that is, $|\lambda|<1$ holds for any eigenvalue $\lambda$ of $J\left(q_{A}, q_{B}, I_{1}, I_{2}\right)$. An equilibrium $\left(q_{A}, q_{B}, I_{1}, I_{2}\right)$ will be unstable if there is an eigenvalue $\lambda$ of $J\left(q_{A}, q_{B}, I_{1}, I_{2}\right)$ such that $|\lambda|>1$.

Proposition 1. The boundary equilibrium $E_{0}$ is an unstable equilibrium.
Proof. Taking the expression of equilibrium $E_{0}$ into (14), we get the Jacobian matrix at $E_{0}$ as follows:

$$
J\left(E_{0}\right)=\left(\begin{array}{cccc}
1+\psi \gamma_{A} & 0 & 0 & 0 \\
0 & 1+\psi \gamma_{B} & 0 & 0 \\
1 & 0 & 0 & 0 \\
0 & 1 & 0 & 0
\end{array}\right)
$$

which has four eigenvalues: $\lambda_{1}=1+\psi \gamma_{A}, \lambda_{2}=1+\psi \gamma_{B}, \lambda_{3}=0$, and $\lambda_{4}=0$. Because $\psi, \gamma_{A}$, and $\gamma_{B}$ are positive parameters, it can be seen that $\lambda_{1}, \lambda_{2}>1$ does not satisfy $E_{0}$ point stability condition. So equilibrium $E_{0}$ is unstable.

Proposition 2. The boundary equilibriums $E_{1}$ and $E_{2}$ are both unstable.

Proof. At the boundary equilibrium point $E_{1}$, the Jacobian matrix (see (14)) is given by 


$$
J\left(E_{1}\right)=\left(\begin{array}{cccc}
1+\gamma_{A}\left(\frac{b \psi+\psi c-c \beta}{b+c}\right) & 0 & 0 & 0 \\
-\frac{\beta \gamma_{B} \omega c}{b+c} & 1-2 \beta \gamma_{B} \omega & -\frac{\beta c \gamma_{B}(1-\omega)}{b+c} & -2 \beta \gamma_{B}(1-\omega) \\
1 & 0 & 0 & 0 \\
0 & 1 & 0 & 0
\end{array}\right) .
$$

By simple calculation, we get four eigenvalues of the matrix $J\left(E_{1}\right)$ :

$$
\begin{aligned}
\lambda_{1} & =0, \\
\lambda_{2} & =1+\gamma_{A}\left(\frac{b \psi+\psi c-c \beta}{b+c}\right), \\
\lambda_{3,4} & \\
& =\frac{1}{2}\left(1-\beta \gamma_{B} \omega \pm \sqrt{1-4 \beta \gamma_{B}+2 \beta \gamma_{B} \omega+\beta^{2} \gamma_{B}^{2} \omega^{2}}\right) .
\end{aligned}
$$

Obviously $\lambda_{2}>1$, so equilibrium $E_{1}$ is unstable. A similar approach shows that $E_{2}$ is unstable too.
3.2. Stability of the Interior Equilibrium. Now we consider the asymptotical stability of the interior equilibrium $E^{*}$. The Jacobian matrix $J$ at $E^{*}=\left(q_{A}^{*}, q_{B}^{*}, I_{1}^{*}, I_{2}^{*}\right)$ takes its formats

$$
J\left(E^{*}\right)=\left(\begin{array}{cccc}
1-\gamma_{A} \omega K_{1} & -2 c \gamma_{A} q_{A}^{*} \omega & -2 \gamma_{A} q_{A}^{*}(1-\omega)(a+c) & 2 c \gamma_{A} q_{A}^{*}(1-\omega) \\
-2 c \gamma_{B} q_{B}^{*} \omega & 1-\gamma_{B} \omega K_{2} & -2 c \gamma_{B} q_{B}^{*}(1-\omega) & -2 \gamma_{B} q_{B}^{*}(1-\omega)(b+c) \\
1 & 0 & 0 & 0 \\
0 & 1 & 0 & 0
\end{array}\right)
$$

where

$$
\begin{aligned}
& K_{1}=2 c q_{A}^{*}+\frac{(a \psi b+\psi c a-c \beta a)}{(b c+a b+a c)}, \\
& K_{2}=2 c q_{B}^{*}+\frac{(a \beta b+\beta c b-\psi b c)}{(b c+a b+a c)} .
\end{aligned}
$$

If $f(\lambda)$ denotes the characteristic polynomial of the Jacobian matrix $J\left(E^{*}\right)$, then

$$
f(\lambda)=\lambda^{4}+g_{1} \lambda^{3}+g_{2} \lambda^{2}+g_{3} \lambda+g_{4} .
$$

By calculation, we get

$$
\begin{aligned}
g_{1} & =\frac{1}{Z}\left[-2 Z+\left((a+c) \gamma_{A} X+(b+c) \gamma_{B} Y\right) \omega\right], \\
g_{2} & =\frac{1}{Z}\left[Z+\left((a+c) \gamma_{A} X+(b+c) \gamma_{B} Y\right)(1-2 \omega)\right. \\
& \left.+X Y \gamma_{A} \gamma_{B} \omega^{2}\right] \\
g_{3} & =\frac{1}{Z}(\omega-1)\left[(a+c) \gamma_{A} X+(b+c) \gamma_{B} Y\right. \\
& \left.-2 X Y \gamma_{A} \gamma_{B} \omega\right], \\
g_{4} & =\frac{1}{Z} X Y \gamma_{A} \gamma_{B}(1-\omega)^{2},
\end{aligned}
$$

where $Z=a b+a c+b c, X=\psi b+\psi c-\beta c$, and $Y=\beta a-\psi c+\beta c$.

For all the roots of the polynomial $f(\lambda)$ (the eigenvalues of the Jacobian matrix $J\left(E^{*}\right)$ ) to lie inside the unit disk, Schur-Cohn Criterion [27] gives the necessary and sufficient conditions as

(i) $f(1)>0$;

(ii) $(-1)^{4} f(-1)>0$;

(iii) the determinants of the $1 \times 1$ matrices $M_{1}^{ \pm}$and the $3 \times 3$ matrices $M_{3}^{ \pm}$are all positive, where

$$
\begin{aligned}
& M_{1}^{ \pm}=1+g_{4}, \\
& M_{3}^{ \pm}=\left(\begin{array}{lll}
1 & \\
g_{1} & 1 & \\
g_{2} & g_{1} & 1
\end{array}\right) \pm\left(\begin{array}{lll}
0 & 0 & g_{4} \\
0 & g_{4} & g_{3} \\
g_{4} & g_{3} & g_{2}
\end{array}\right) .
\end{aligned}
$$

In our model, we have $f(1)=1+g_{1}+g_{2}+g_{3}+$ $g_{4}=X Y \gamma_{A} \gamma_{B} / Z, 1 \pm g_{4}=1 \pm(1 / Z) X Y \gamma_{A} \gamma_{B}(1-\omega)^{2}$; then, from the nonnegativity conditions (13a) and (13b), we know that the first condition $f(\lambda)>0$ and $1 \pm g_{4}>0$ must hold. Consequently, we conclude that the interior equilibrium point $E^{*}$ of system (10) is asymptotically locally stable if it meets the following conditions: 


$$
\begin{aligned}
f(-1) & =1-g_{1}+g_{2}-g_{3}+g_{4}=\frac{4 Z+2\left[(a+c) X \gamma_{A}+(b+c) Y \gamma_{B}\right](1-2 \omega)-X Y \gamma_{A} \gamma_{B}(2 \omega-1)^{2}}{Z}>0, \\
\operatorname{Det}\left(M_{3}^{ \pm}\right) & >0,
\end{aligned}
$$

where $\operatorname{Det}(M)$ represents the determinant of matrix $M$.

\section{Numerical Simulation}

In this section, numerical simulations show how the system evolves under different levels of parameters, especially with adjustment speeds $\gamma_{A}$ and $\gamma_{B}$. In all the numerical simulations, the other parameters are fixed: $a=1, b=0.6, \omega=0.6$, $c=0.4, \psi=5$, and $\beta=4$.

So, we will use some numerical simulations to show the complicated behavior of the model (stability, period-doubling bifurcation, and chaos).

Figure 1 shows the bifurcation diagram of quantities $q_{A}$ and $q_{B}$ with the adjustment speed $\gamma_{A}$ while the other parameters are constant and have taken the value $\gamma_{B}=0.6$. This figure shows that the equilibrium points $q_{A}$ and $q_{B}$ are locally stable for $\gamma_{A}<0.0755$.

Figure 2 shows the bifurcation diagram of the quantities $q_{A}$ and $q_{B}$ with the adjustment speed $\gamma_{B}$ while the other parameters are constant and have taken the value $\gamma_{A}=0.5$. This figure shows that the equilibrium points $q_{A}$ and $q_{B}$ are locally stable for $\gamma_{B}<0.443$, and such a complicated process (period-doubling bifurcation, Neimark-Sacker bifurcation, periodic window, etc.) continues to lead the system to chaos.

The observations from Figures 1 and 2 show that when $\gamma_{A}$ and $\gamma_{B}$ are increasing, it shows more complex dynamic phenomena by appearing in the process of the evolution of Neimark-Sacker bifurcation and cycle window.

In Figure 3, the nonlinear dynamic system (10) can be showed from three dimensions of strange attractor, and strange attractor shows in the system space which is comprised by three arbitrary state variables.

Figure 4 shows the sensitivity of system (when loosing stability) to initial conditions, with $\left(q_{A 1}(0), q_{B 1}(0), I_{1}(0)\right.$, $\left.I_{2}(0)\right)=(1,2,1,2)$ and $\left(q_{A 1}(0)+0.0001, q_{B 1}(0), I_{1}(0), I_{2}(0)\right)$. Figure 4 shows the difference among the different orbits with slightly deviated initial values which builds up rapidly after a number of iterations, although their initial states are indistinguishable.

In Figures 5(a), 5(b), and 5(c), the bifurcation diagrams are plotted for the weight coefficient assigned $w$ when $\gamma_{B}$ is fixed as 0.6. Figure 5(a) is for $\gamma_{A}=0.105$, Figure 5(b) is for $\gamma_{A}=0.515$, and Figure 5(c) is for $\gamma_{A}=0.615$. Figure 5(a) shows a reverse period-doubling bifurcation, while Figures 5(b) and 5(c) combine reverse period-doubling bifurcation and reverse Neimark-Sacker bifurcation. The three figures show that the system tends toward stability with the increased weight coefficient, which also shows that a high residual rate has a positive effect on the system stability.

\section{Chaos Control}

From the numerical simulations, the adjustment rate and the weight coefficient have great influence on the stability of system (10). If the model parameters fail to locate into the stable region required, the behaviors of the dynamics will be much complicated. In a real economic system, chaos is not desirable and will be not expected, and it is needed to be avoided or controlled so that the dynamic system would work better. In this section, we use the time-delayed feedback control (e.g., $[18,28])$ to control system chaos. We modify the first equation of system (10) by intercalating a controller $k\left(q_{A 1}(t)-q_{A 1}(t+1)\right)$ as a small perturbation, where $k>0$ is a controlling coefficient. Then the controlled system is given by

$$
\begin{aligned}
& q_{A}(t+1)=q_{A}(t)+\gamma_{A} q_{A}(t) \\
& \cdot\left[\omega\left(\psi-2(a+c) q_{A}(t)-2 c q_{B}(t)\right)\right. \\
& \left.+(1-\omega)\left(\psi-2(a+c) I_{1}(t)-2 c I_{2}(t)\right)\right] \\
& \quad+k\left(q_{A}(t)-q_{A}(t+1)\right), \\
& q_{B}(t+1)=q_{B}(t)+\gamma_{B} q_{B}(t) \\
& \quad \cdot\left[\omega\left(\beta-2(b+c) q_{B}(t)-2 c q_{A}(t)\right)\right. \\
& \left.\quad+(1-\omega)\left(\beta-2(b+c) I_{2}(t)-2 c I_{1}(t)\right)\right] \\
& I_{1}(t+1)=q_{A}(t) \\
& I_{2}(t+1)=q_{B}(t) .
\end{aligned}
$$

It is easy to see that the new system (25) has the same equilibriums as system (10) and it takes the following equivalent form:

$$
\begin{aligned}
q_{A}(t+1)=q_{A}(t)+\frac{1}{1+k} \gamma_{A} q_{A}(t) \\
\cdot\left[\omega\left(\psi-2(a+c) q_{A}(t)-2 c q_{B}(t)\right)\right. \\
\left.+(1-\omega)\left(\psi-2(a+c) I_{1}(t)-2 c I_{2}(t)\right)\right], \\
q_{B}(t+1)=q_{B}(t)+\gamma_{B} q_{B}(t) \\
\cdot\left[\omega\left(\beta-2(b+c) q_{B}(t)-2 c q_{A}(t)\right)\right. \\
\left.+(1-\omega)\left(\beta-2(b+c) I_{2}(t)-2 c I_{1}(t)\right)\right] \\
I_{1}(t+1)=q_{A}(t) \\
I_{2}(t+1)=q_{B}(t) .
\end{aligned}
$$




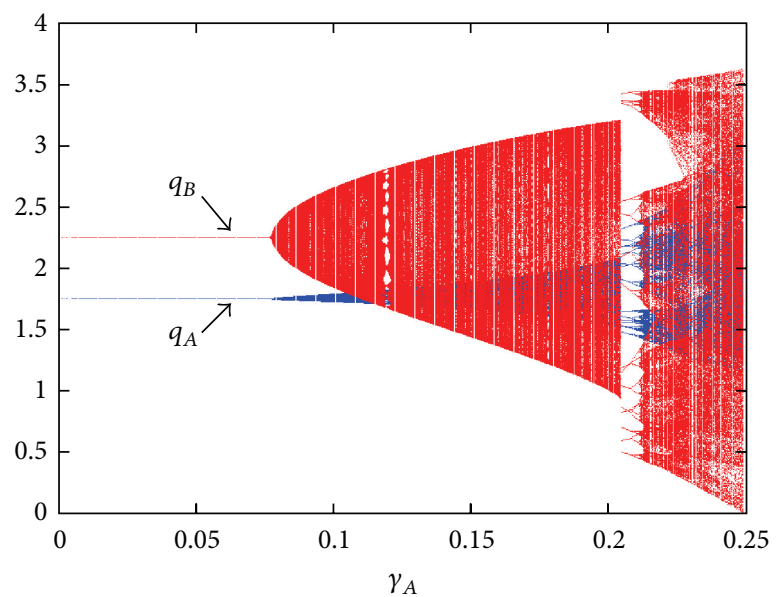

FIGURE 1: Bifurcation diagram for the quantities $q_{A}$ and $q_{B}$ with the adjustment speed $\gamma_{A}$.

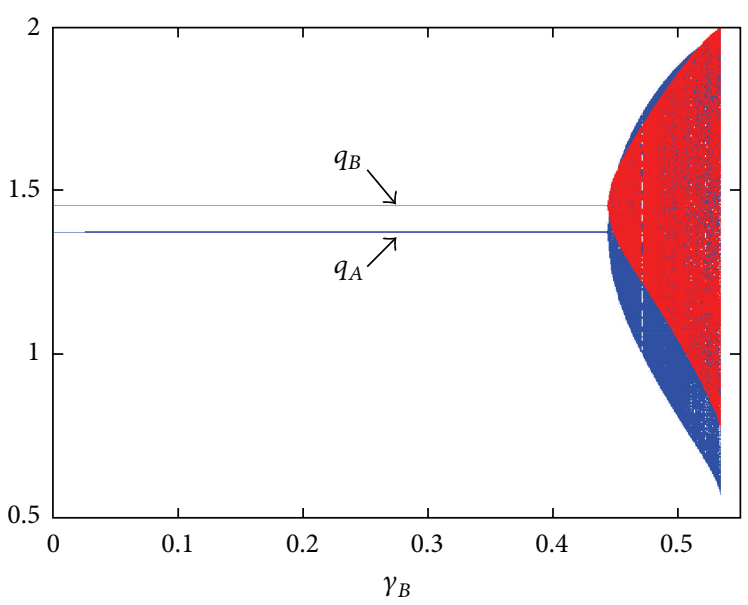

FIGURE 2: Bifurcation diagram for the quantities $q_{A}$ and $q_{B}$ with the adjustment speed $\gamma_{B}$.

The Jacobian matrix of the controlled system (26) is given by

$$
J=\left(\begin{array}{cccc}
1+\frac{\gamma_{A} N_{1}}{1+k} & -\frac{2 c \omega \gamma_{A} q_{A}(t)}{1+k} & -\frac{2(1-\omega)(a+c) \gamma_{A} q_{A}(t)}{1+k} & -\frac{2 c \gamma_{A} q_{A}(t)(1-\omega)}{1+k} \\
-2 \omega c \gamma_{B} q_{B}(t) & 1+\gamma_{B} N_{2} & -2 c \gamma_{B} q_{B}(t)(1-\omega) & -2(b+c) \gamma_{B} q_{B}(t)(1-\omega) \\
1 & 0 & 0 & 0 \\
0 & 1 & 0 & 0
\end{array}\right)
$$

where

$$
\begin{aligned}
N_{1}= & \omega\left(\psi-4(a+c) q_{A 1}(t)-2 c q_{B 1}(t)\right) \\
& +(1-\omega)\left(\psi-2(a+c) I_{1}(t)-2 c I_{2}(t)\right), \\
N_{2}= & \omega\left(\beta-4(b+c) q_{B 1}(t)-2 c q_{A 1}(t)\right) \\
& +(1-\omega)\left(\beta-2(b+c) I_{2}(t)-2 c I_{1}(t)\right) .
\end{aligned}
$$

As has been shown in Figures 1 and 2, chaotic behavior of system (10) occurs when all the model parameters take their values as $\left(a, b, c, \psi, \beta, \gamma_{A}, \gamma_{B}, \omega\right)=(1,0.6,0.4,5$, $4,0.55,0.6,0.55)$.

Using this group of parameters values, we obtain the Jacobian matrix (see (26)) at the interior equilibrium as follows.

As shown in Figure 1, the controlled system near the adjustment coefficient $\gamma_{A}=0.081$ is entered into a state of chaos. When the model parameter selection, the initial 


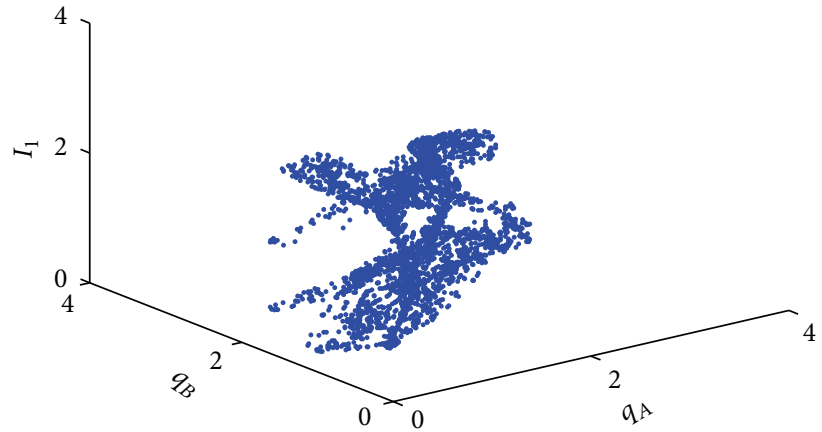

(a)

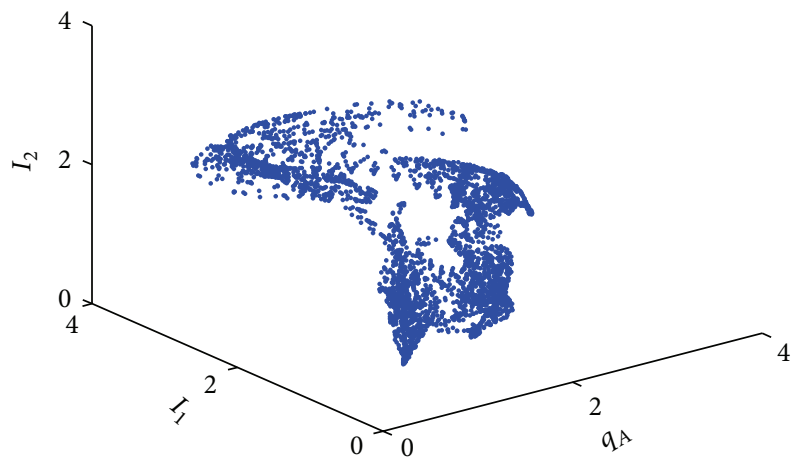

(c)

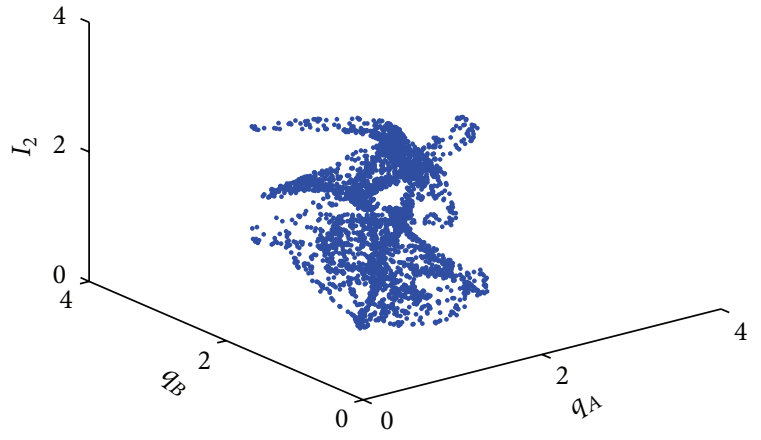

(b)

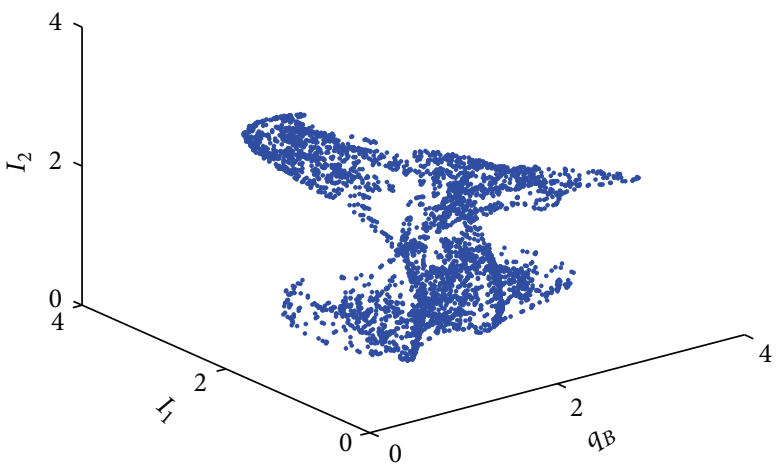

(d)

Figure 3: The three-dimensional strange attractors of the dynamical system (10).

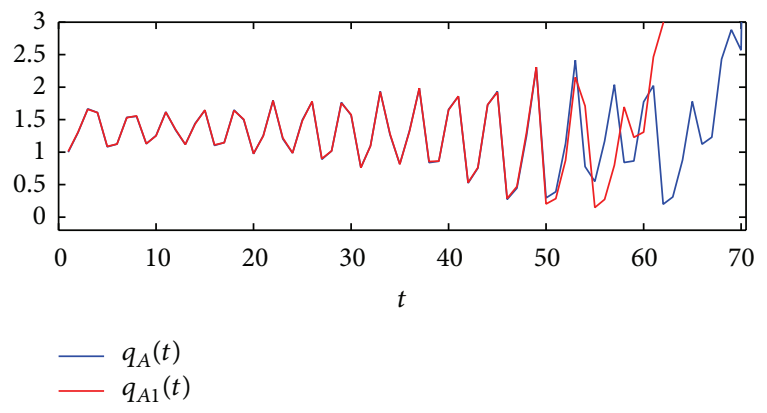

(a)

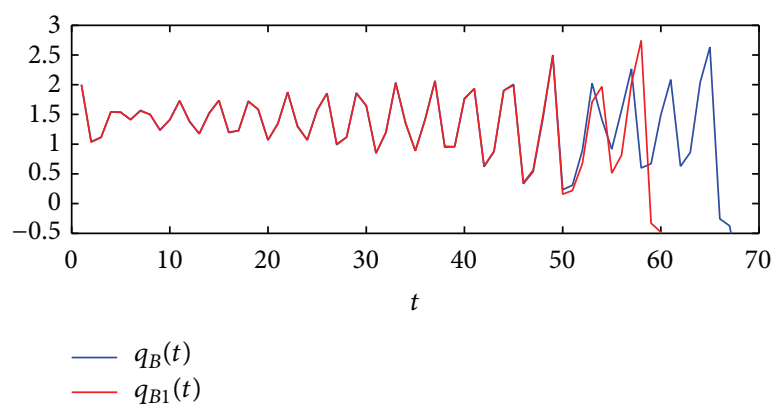

(b)

FIGURE 4: Sensitive dependence for the dynamical system (10) on initial conditions. conditions $\left(q_{A 1}(0), q_{B 1}(0), I_{1}(0), I_{2}(0)\right)=(1,2,1,2)$, and system (10) show the messy chaos phenomenon, the external variable parameter $\gamma_{A}$ is in a stable area. Analysis under the controlled system (26) in this parameter values of the chaos represents the stability of the new system (26). The parameter values in the system use (26) the Jacobian matrix as follows:

$$
J=\left(\begin{array}{cccc}
1+\frac{0.517}{1+k} & -\frac{0.242}{1+k} & -\frac{0.693}{1+k} & -\frac{0.198}{1+k} \\
-0.528 & -2 & -0.432 & -1.08 \\
1 & 0 & 0 & 0 \\
0 & 1 & 0 & 0
\end{array}\right)
$$

Corresponding characteristic polynomial calculates the matrix $P(\lambda)=\lambda^{4}+v_{1} \lambda^{3}+v_{2} \lambda^{2}+v_{3} \lambda+v_{4}$, with the coefficient of theory, as follows:

$$
\begin{aligned}
& v_{1}=\frac{k+0.483}{1+k}, \\
& v_{2}=\frac{-0.92 k-1.38878}{1+k}, \\
& v_{3}=\frac{-1.08 k-0.461448}{1+k}, \\
& v_{4}=\frac{0.662904}{1+k} .
\end{aligned}
$$




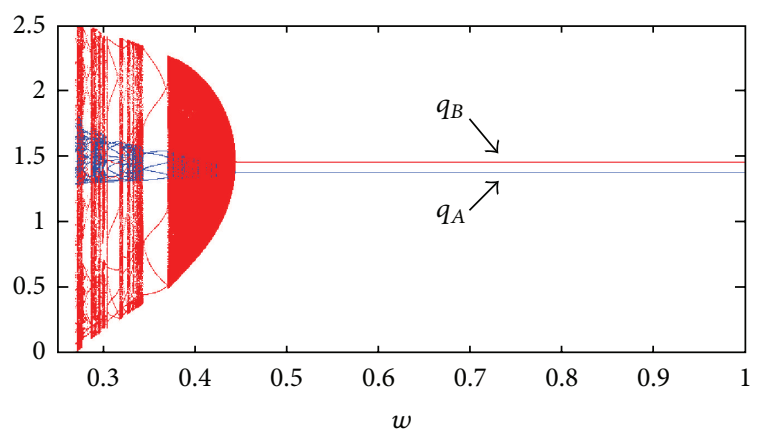

(a)

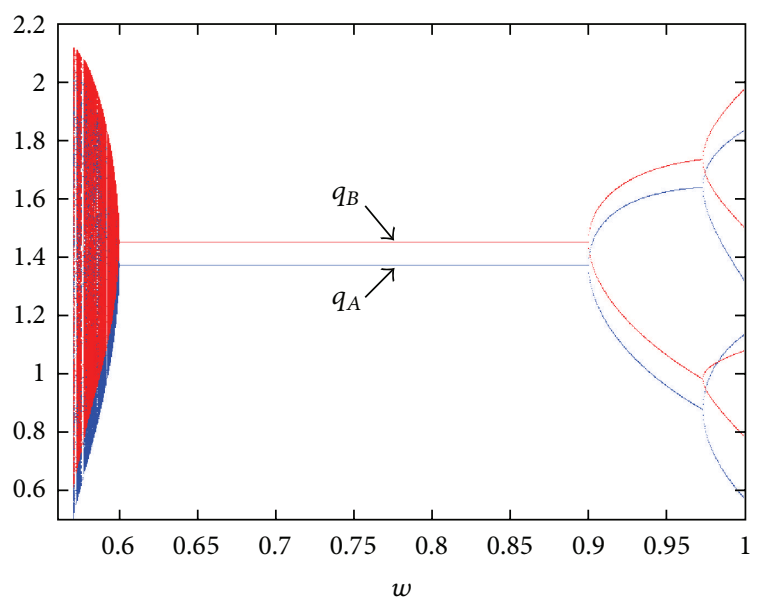

(b)

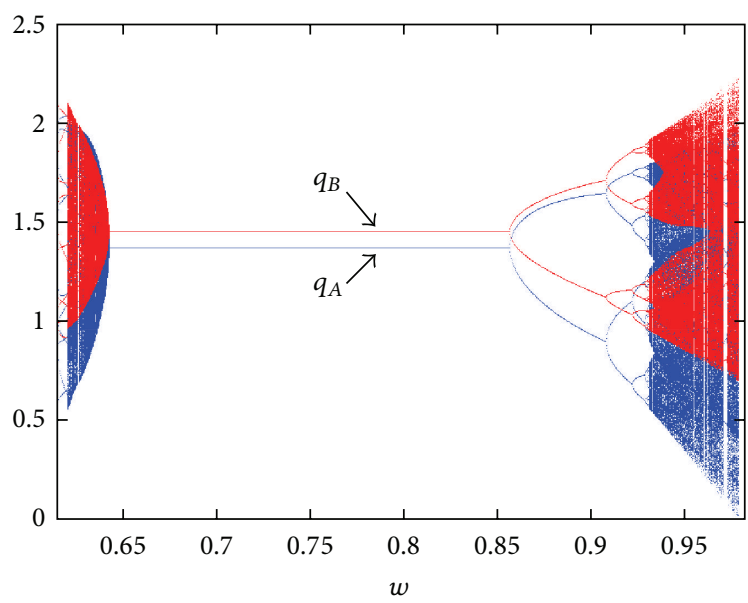

(c)

Figure 5: Bifurcation diagrams of dynamic system (10) with the weight coefficient assigned $w$.

According to Schur-Cohn stability criterion, when the Jacobi matrix of the characteristic polynomial $P(\lambda)$ of the coefficients satisfy the following conditions:

$$
\begin{aligned}
& \left|1+v_{2}+v_{4}\right|>v_{1}+v_{3} \\
& \left|1-v_{4}^{2}+v_{2} v_{4}-v_{2} v_{4}^{2}+v_{1} v_{3} v_{4}-v_{3}^{2}\right| \\
& \quad>v_{2}-v_{2} v_{4}+v_{4}-v_{4}^{3}-v_{1} v_{3}+v_{1}^{2} v_{4},
\end{aligned}
$$

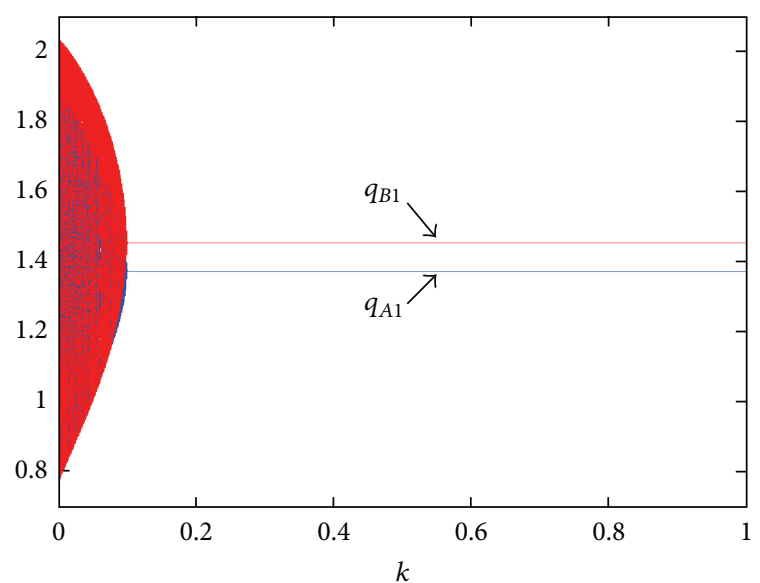

FIGURE 6: Bifurcation diagrams of system (26) with the controlling factor $k$.

the entire characteristic root of the matrix is less than 1 , which shows that dynamic system (26) is to take the set of parameter values under stable, so the controlled system (10) of chaos control tends to be a stable orbit. From the stability conditions, we get that all the eigenvalues of the matrix will lie inside the unit disk by providing $k>0.0917$. When $k>0.0917$, the controlled system (26) will be asymptotically locally stable.

In Figure 6, it is obviously observed that, with the control coefficient $k$ increasing, the system gradually gets out of chaos and periodic windows and achieves stability when $k>$ 0.0917 . When $k=1.5$ and $k=3.5$, Figures 7 and 8 show the stable behaviors of the orbits of the controlled system beginning from the initial state $\left(x_{1}(0), x_{2}(0), I_{1}(0), I_{2}(0)\right)=$ $(1,2,1,2)$. These two graphs can be found in the stable region, the feedback gains strength value, and the more chaotic behavior can quickly control the stable orbit.

\section{Conclusion}

In this study we have taken into consideration a dynamic Cournot game based on quadratic cost function which is built with the same assumption of bounded rationality. We discuss the stability of each equilibrium solution by using the nonlinear system. Numerical simulations are used to provide experimental evidence for the complicated evolution behaviors of the system. It proves that the chaotic behavior of the system can be controlled. It proves that parameters play an important role in the stability of the economy systems based on analysis of dynamical behaviors of the established game models. It shows that the weight coefficient assigned can enhance the stability of systems. If each firm renews its investment strategy too fast or too slow, the systems will postpone converging to equilibrium and may respond intricately, including bifurcation, chaos, and initial state of sensitivity. Meanwhile, time-delayed feedback control could be used to stabilize the chaotic behaviors of the dynamic systems. 


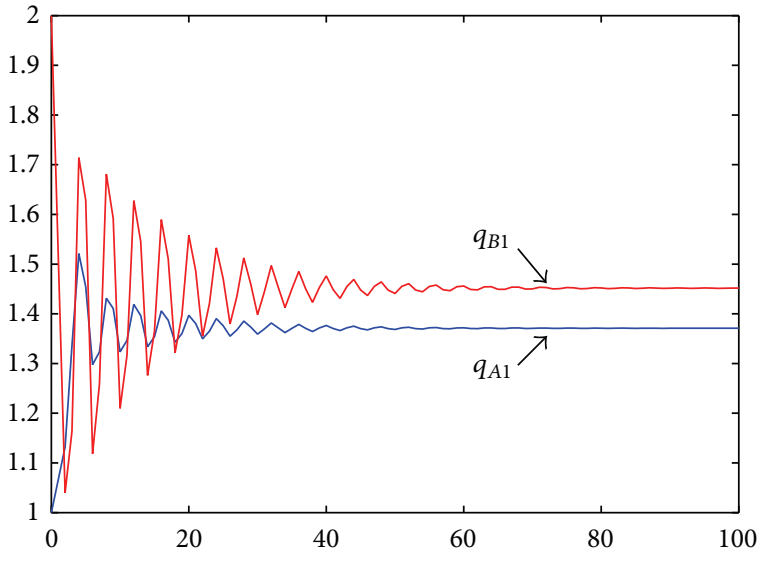

Figure 7: Evolution for the dynamic system (26) with the controlling factor $k=1.5$.

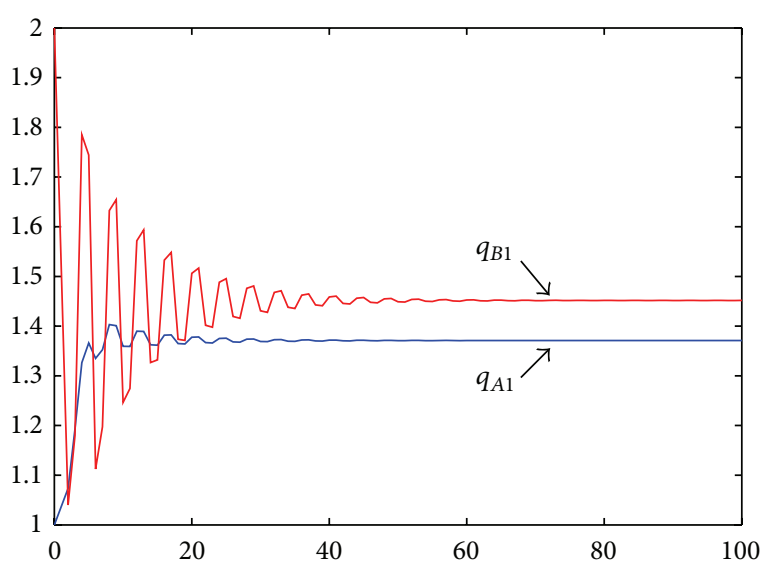

FIGURE 8: Evolution for the dynamic system (26) with the controlling factor $k=3.5$.

\section{Competing Interests}

The author declares that they have no competing interests.

\section{Acknowledgments}

This work is supported by the National Nature Science Foundation of China (nos. 71171099, 71471076, 71001028, 71201071, and 71373103) and China Scholarship Council under Grant 20123227110011.

\section{References}

[1] A. Cournot, Researches into the Mathematical Principles of the Theory of Wealth, 1838, Edited by N. Bacon, Macmillan, New York, NY, USA, 1897.

[2] R. D. Theocharis, "On the stability of the Cournot solution on the oligopoly problem," The Review of Economic Studies, vol. 27, no. 2, pp. 133-134, 1960.

[3] T. Puu, "Complex dynamics with three oligopolists," Chaos, Solitons \& Fractals, vol. 7, no. 12, pp. 2075-2081, 1996.
[4] M. Kopel, "Simple and complex adjustment dynamics in Cournot duopoly models," Chaos, Solitons \& Fractals, vol. 7, no. 12, pp. 2031-2048, 1996.

[5] T. Puu, "The chaotic duopolists revisited," Journal of Economic Behavior \& Organization, vol. 33, no. 3-4, pp. 385-394, 1998.

[6] E. Ahmed and H. N. Agiza, "Dynamics of a cournot game with n-competitors," Chaos, Solitons \& Fractals, vol. 9, no. 9, pp. 15131517, 1998.

[7] H. N. Agiza, "Explicit stability zones for Cournot game with 3 and 4 competitors," Chaos, Solitons \& Fractals, vol. 9, no. 12, pp. 1955-1966, 1998.

[8] A. Agliari, L. Gardini, and T. Puu, "The dynamics of a triopoly Cournot game," Chaos, Solitons \& Fractals, vol. 11, no. 13, pp. 2531-2560, 2000.

[9] J. B. Rosser Jr., "The development of complex oligopoly dynamics theory," in Oligopoly Dynamics: Models and Tools, T. Puu and I. Sushko, Eds., pp. 15-29, Springer, Berlin, Germany, 2002.

[10] G. I. Bischiy and A. Naimzadaz, "Global analysis of a dynamic duopoly game with bounded rationality," in Advances in Dynamic Games and Applications, vol. 5 of Annals of the International Society of Dynamic Games, pp. 361-386, Springer, Berlin, Germany, 1999.

[11] E. Ahmed, H. N. Agiza, and S. Z. Hassan, "On modifications of Puu's dynamical duopoly," Chaos, Solitons \& Fractals, vol. 11, no. 7, pp. 1025-1028, 2000.

[12] H. N. Agiza, A. S. Hegazi, and A. A. Elsadany, "The dynamics of Bowley's model with bounded rationality," Chaos, Solitons \& Fractals, vol. 12, no. 9, pp. 1705-1717, 2001.

[13] H. N. Agiza, A. S. Hegazi, and A. A. Elsadany, "Complex dynamics and synchronization of a duopoly game with bounded rationality," Mathematics and Computers in Simulation, vol. 58, no. 2, pp. 133-146, 2002.

[14] H. N. Agiza and A. A. Elsadany, "Nonlinear dynamics in the Cournot duopoly game with heterogeneous players," Physica A: Statistical Mechanics and Its Applications, vol. 320, pp. 512-524, 2003.

[15] H. N. Agiza and A. A. Elsadany, "Chaotic dynamics in nonlinear duopoly game with heterogeneous players," Applied Mathematics and Computation, vol. 149, no. 3, pp. 843-860, 2004.

[16] J. X. Zhang, Q. L. Da, and Y. H. Wang, "Analysis of nonlinear duopoly game with heterogeneous players," Economic Modelling, vol. 24, no. 1, pp. 138-148, 2007.

[17] Z. W. Ding, Q. L. Hang, and L. X. Tian, "Analysis of the dynamics of Cournot team-game with heterogeneous players," Applied Mathematics and Computation, vol. 215, no. 3, pp. 1098$1105,2009$.

[18] E. M. Elabbasy, H. N. Agiza, and A. A. Elsadany, "Analysis of nonlinear triopoly game with heterogeneous players," Computers \& Mathematics with Applications, vol. 57, no. 3, pp. 488-499, 2009.

[19] T. Dubiel-Teleszynski, "Nonlinear dynamics in a heterogeneous duopoly game with adjusting players and diseconomies of scale," Communications in Nonlinear Science and Numerical Simulation, vol. 16, no. 1, pp. 296-308, 2011.

[20] Y. Fan, T. Xie, and J. Du, "Complex dynamics of duopoly game with heterogeneous players: a further analysis of the output model," Applied Mathematics and Computation, vol. 218, no. 15, pp. 7829-7838, 2012.

[21] A. L. Bowley, The Mathematical Groundwork of Economics, Oxford University Press, Oxford, UK, 1924. 
[22] S. Chakrabarti and H. Haller, "An analysis of advertising wars," Manchester School, vol. 79, no. 1, pp. 100-124, 2011.

[23] A. Dixit, "A model of duopoly suggesting a theory of entry barriers," The Bell Journal of Economics, vol. 10, no. 1, pp. 20-32, 1979.

[24] M. Spence, "Product differentiation and welfare," American Economic Review, vol. 66, pp. 407-414, 1976.

[25] P. Billand, C. Bravard, S. Chakrabarti, and S. Sarangi, Networks of Collaboration in Multi-market Oligopolies, 2010, http://mpra .ub.uni-muenchen.de/28188.

[26] Z. W. Ding, Q. Wang, and S. M. Jiang, "Analysis on the dynamics of a Cournot investment game with bounded rationality," Economic Modelling, vol. 39, pp. 204-212, 2014.

[27] S. N. Elaydi, An Introduction to Difference Equations, Springer, New York, NY, USA, 2005.

[28] J. A. Holyst and K. Urbanowicz, "Chaos control in economical model by time-delayed feedback method," Physica A: Statistical Mechanics and Its Applications, vol. 287, no. 3-4, pp. 587-598, 2000. 


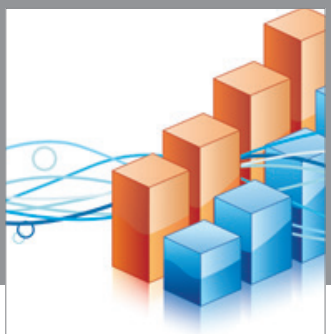

Advances in

Operations Research

vatem alat4

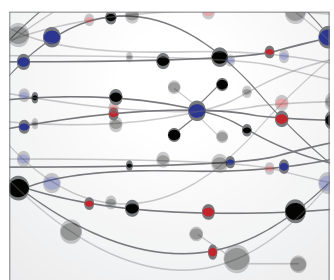

\section{The Scientific} World Journal
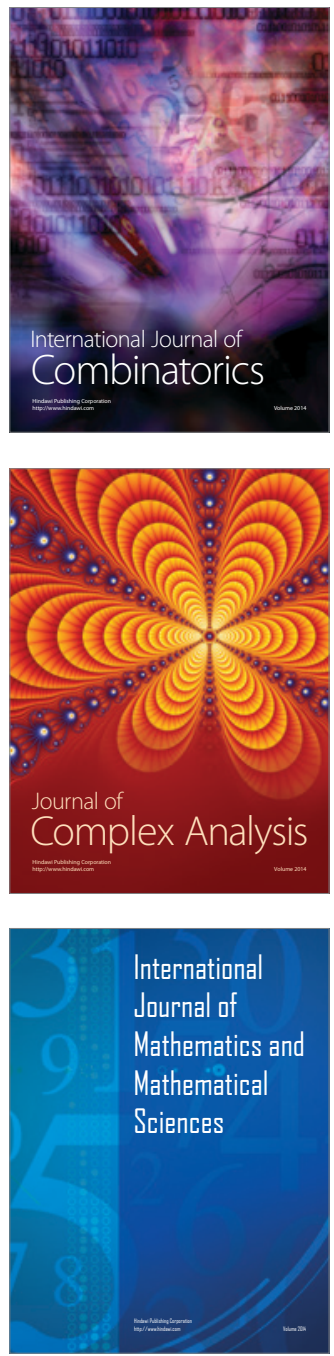
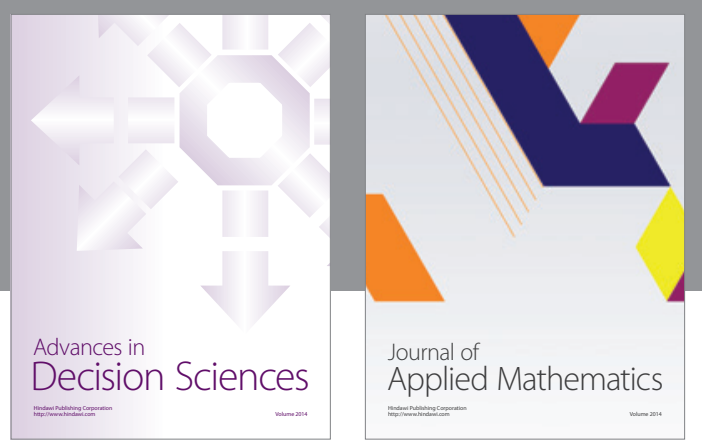

Algebra

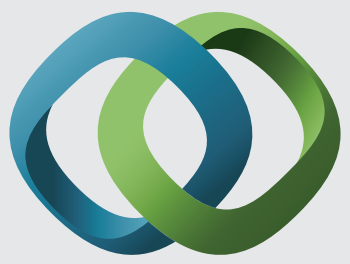

\section{Hindawi}

Submit your manuscripts at

http://www.hindawi.com
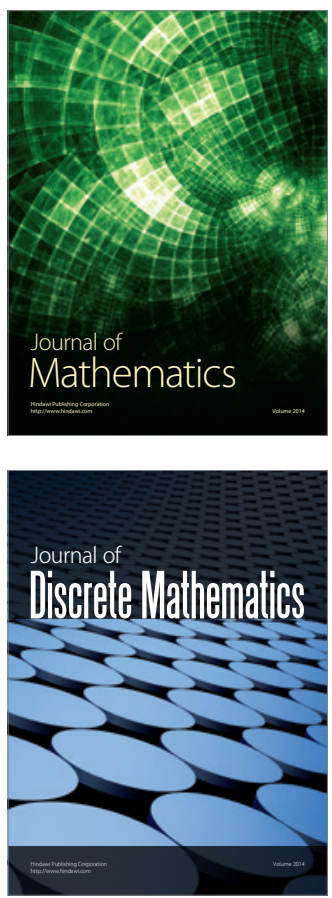

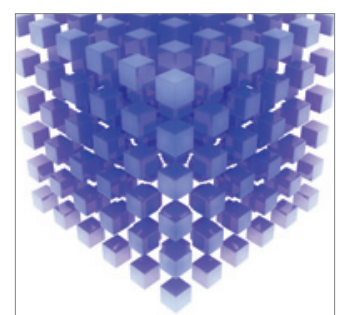

Mathematical Problems in Engineering
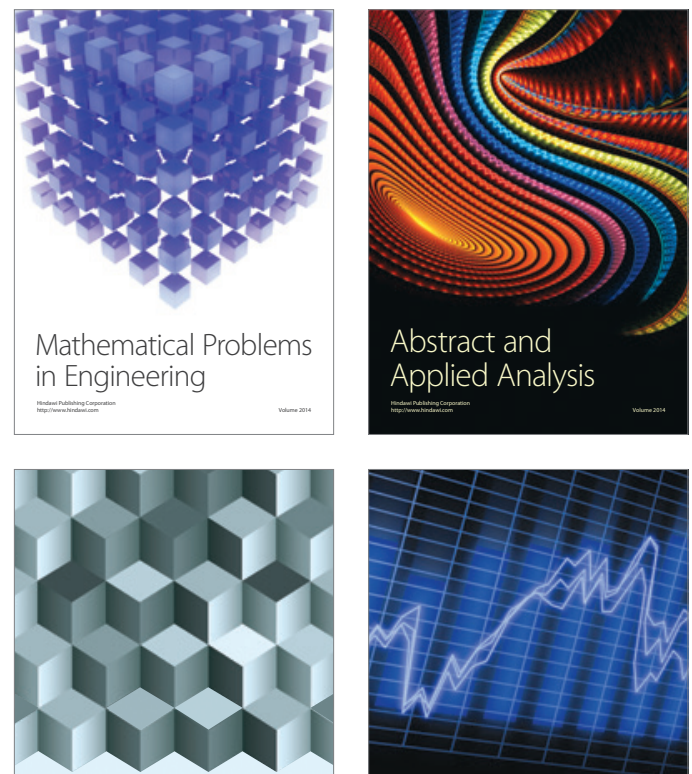

Journal of

Function Spaces

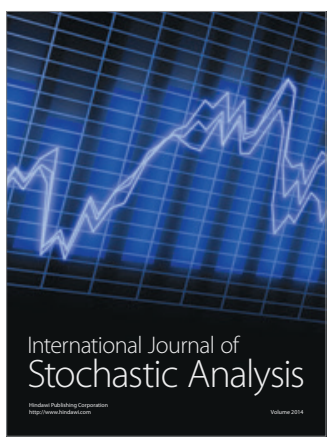

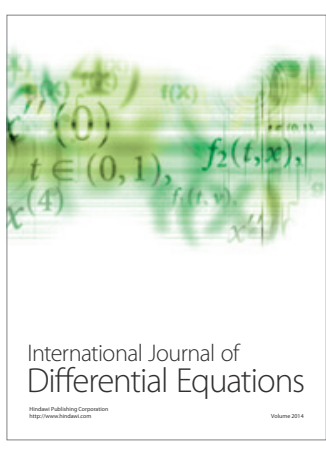
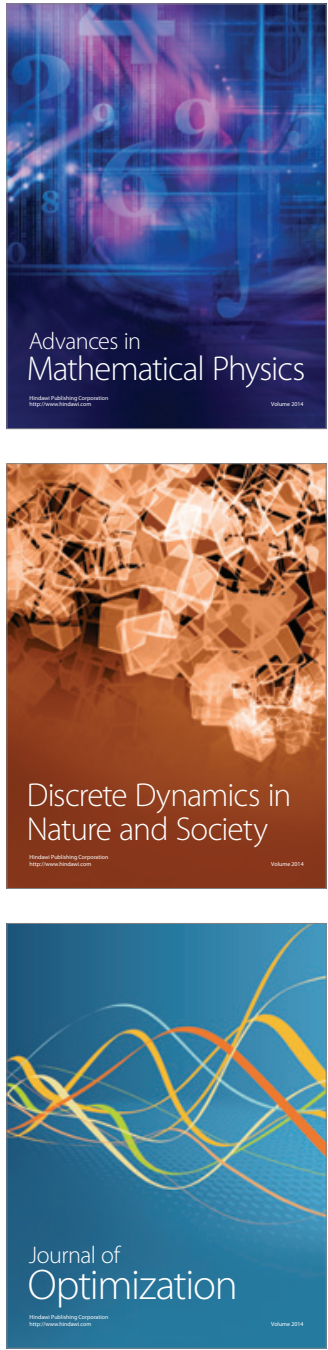